SECTION 31. Economic research, finance, innovation, risk management.

Anatoly Aleksandrovich Naumov

Docent, Candidate of Technical Sciences, corresponding member of International Academy of Theoretical \& Applied Sciences Center of Applied Mathematical Research, Novosibirsk, Russia,

E-mail: a_a_naumov@mail.ru

Sapargali Utepovich Zhanatauov Candidate of Physic and mathematical Sciences, senior research associate, Docent of Department Engineering and Informatics, Central Asian Technological University, Almaty, Kazakhstan E-mail: sapagtu@mail.ru

\title{
FUZZY SETS IN INVESTMENT PROJECTS RISK ESTIMATION
}

Abstract: The paper discusses the approaches to the problems of investment projects risk analysis using the uncertainty in the knowledge of the membership function of the fuzzy sets.

Keywords: Risks, investment project, fuzzy sets, membership function.

\section{УДК 330.322 .5}

\section{ИСПОЛЬЗОВАНИЕ РАЗМЫТЫХ МНОЖЕСТВ ПРИ ОЦЕНИВАНИИ РИСКОВ ИНВЕСТИЦИОННЫХ ПРОЕКТОВ}

Аннотация: В работе рассмотрены подходы к решению задач анализа рисков инвестиционных проектов при использовании неопределенности в знании функиии принадлежности размытых множеств.

Ключевые слова: Риски, инвестиционный проект, размытые множества, функиия принадлежности.

Обычно при оценивании рисков инвестиционных проектов (ИП) используются методы имитационного моделирования с учетом функции неопределенности значений параметров проекта. В качестве таких параметров обычно выступают элементы входного и выходного потоков проекта, ставки заемного процента, уровень инфляции и пр. А в качестве характеристики неопределенности используются функции распределения (или функции плотности) вероятностей для этих параметров. Если функции распределения заданы неточно (т.е. присутствует неопределенность в их задании), то обычно применяют аналогичные функции, построенные на размытых множествах [1-9]. В данной работе предполагается, что и размытая функция плотности вероятностей параметров проекта известна неточно.

Риски инвестиционных проектов (ИП), представимых бизнес-процессами (в виде потоковых моделей) $\overparen{B P}_{s}(t)[10]$, обусловлены неопределенностями в знании значений характеристик бизнес-процессов ВР и их параметров $\pi$. Условимся бизнеспроцессы с неопределенностями обозначать через $\widetilde{B P}=\left\{\widetilde{B P}_{i}(t)\right\}, i=1,2, \ldots, N, \quad$ и, соответственно, их параметры - через $\tilde{\pi}=\left\{\tilde{\pi}_{i}\right\}, i=1,2, \ldots, N$. Очевидно, такая неопределенность в знании характеристик ИП (и соответствующих им бизнес- 
процессов) приводит к тому, что вместо вектора показателей эффективности (номинального, планового) $\vec{Q}$ (или $\left\{\vec{Q}_{(i)}\right\}, i=1,2, \ldots, N$ ) реально будем иметь дело с вектором $\overrightarrow{\widetilde{Q}}$ (или с векторами $\left\{\overrightarrow{\widetilde{Q}}_{(i)}\right\}, i=1,2, \ldots, N$ ). Величина отклонения вектора $\vec{Q}$ от вектора $\overrightarrow{\widetilde{Q}}$ и будет характеризовать риск ИП. Процедура оценивания рисков (в динамике - оценивание вектора рисков $\vec{R}(t))$ может быть условно представлена отображением: $\langle\widetilde{B P}, \tilde{\pi}\rangle \stackrel{\varphi_{R}}{\longrightarrow} \vec{R}(t)$, где $\varphi_{R}$ - оператор оценивания рисков. Оператор $\varphi_{R}$ оценивает отклонения между векторами $\vec{Q}(t)$ и $\overrightarrow{\widetilde{Q}}(t)$.

Индивидуальными рисками можно воспользоваться, если объединить их в пары с самими показателями, например, такого вида: $\left\langle Q_{i, \text { пот }}, R_{j, i}\right\rangle, i \in\{1,2, \ldots, M\}$, где $j-$ номер (индекс) вида риска, $M-$ число показателей эффективности, или $\left\langle Q_{i, \text { пот }} ;\left\{R_{j, i}\right\}, j=1,2, \ldots, K\right\rangle, \quad i \in\{1,2, \ldots, M\}$, в случае, если каждому из показателей сопоставить множество оценок рисков $\left\{R_{j, i}\right\}, j=1,2, \ldots, K$, ему соответствующих. Такие пары порождают характеристики ИП вида $\vec{Q}_{R}=\left(Q_{1 R}, Q_{2 R}, \ldots, Q_{M R}\right)^{T}$, где $Q_{i R}=\left\langle Q_{i, \text { ном }} ;\left\{R_{j, i}\right\}, j=1,2, \ldots, K\right\rangle, \quad i \in\{1,2, \ldots, M\}$.

Предположим, что при моделировании и оценивании значений показателей $\overrightarrow{\widetilde{Q}}(t)$ используются характеристики: 1) $f(\tilde{\pi})$ - плотность вероятностей возмущенных значений параметров $\pi$ ИП; 2) $\mu_{A}(\tilde{\pi}) \cdot f(\tilde{\pi})$ - плотность вероятностей для размытого множества параметров. Здесь $\mu_{A}(\tilde{\pi})$ - функция принадлежности [1-3], $A \subseteq$ П, Побласть допустимых значений параметров $\tilde{\pi}$. Часто предполагается, что переход от функции $f(\tilde{\pi})$ к функции $\mu_{A}(\tilde{\pi}) \cdot f(\tilde{\pi})$ снимает многие вопросы, связанные с незнанием точного вида классической функции плотности вероятностей. Однако, на наш взгляд, нахождение точного значения функции принадлежности является задачей не менее простой, чем нахождение функции $f(\tilde{\pi})$. Здесь предлагается неопределенность в знании точного вида этих функций представить в виде множеств их допустимых значений: $f(\tilde{\pi}) \in \mathrm{F}_{f}(\tilde{\pi})$ и $\mu_{\dot{A}}(\tilde{\pi}) \in \mathrm{F}_{\mu}(\tilde{\pi})$. Например, множества $\mathrm{F}_{f}(\tilde{\pi})$ и $\mathrm{F}_{\mu}(\tilde{\pi}) \quad$ могут $\quad$ быть дискретными $\quad\left(\mathrm{F}_{f, N_{f}}(\tilde{\pi})=\left\{f_{1}(\tilde{\pi}), f_{2}(\tilde{\pi}), \ldots, f_{N_{f}}(\tilde{\pi})\right\}\right) \quad$ или параметризованными так, что при изменении параметров элементы этих множеств принимают все допустимые значения из области неопределенности:

$$
\mathrm{F}_{\mu, \theta}(\tilde{\pi})=\left\{\mu_{A}(\tilde{\pi}, \theta) \mid \theta \in \Theta\right\} .
$$

Тогда, в результате моделирования в соответствии с множествами функций неопределенности $\mathrm{F}_{f, N_{f}}(\tilde{\pi})$ или $\quad \mathrm{F}_{\mu, \theta}(\tilde{\pi})$ будут получены пары «показатели эффективности - риски» вида: $Q_{i R}=\left\langle Q_{i, н о м} ;\left\{R_{j, i}^{(l)}\right\}, j=1,2, \ldots, K, l=1,2, \ldots, N_{f}\right\rangle$, $i \in\{1,2, \ldots, M\}$ для $\mathrm{F}_{f, N_{f}}(\tilde{\pi})$ и $Q_{i R}=\left\langle Q_{i, \text { ном }} ;\left\{R_{j, i}(\theta)\right\}, j=1,2, \ldots, K, \theta \in \Theta\right\rangle, \quad i \in\{1,2, \ldots, M\}$ для $\mathrm{F}_{\mu, \theta}(\tilde{\pi})$. Остается только свернуть множество рисков, оцененных для показателей эффективности, в одно значение. Например, если риски оцениваются наибольшими 
возможными потерями, то свертки могут иметь такой вид: $R_{j_{0}, i}^{(\max )}=\max _{l=1,2, \ldots, N_{f}} R_{j_{0}, i}^{(l)}$ для $\mathrm{F}_{f, N_{f}}(\tilde{\pi}) \quad$ и $\quad R_{j_{0}, i}^{(\max )}=\max _{\theta \in \Theta} R_{j_{0}, i}(\theta) \quad$ для $\mathrm{F}_{\mu, \theta}(\tilde{\pi})$. И окончательно получим оценки: $Q_{i R}=\left\langle Q_{i, \text { ном }} ;\left\{R_{j_{0}, i}^{(\max )}\right\}\right\rangle, \quad i \in\{1,2, \ldots, M\} . \quad$ Здесь $\quad$ значение индекса $j_{0} \in\{1,2, \ldots, K\}$ фиксировано и соответствует риску наибольших возможных потерь.

Приведем пример оценивания рисков показателей аутсорсингового кластера (АК) [10]. Для этого будем оценивать два показателя $N F V_{(l)}$ (дохода) и $I R R_{N F V_{(l)}}$ (доходности). Предположим, что отклонения от запланированных (номинальных, плановых) значений потоков АК подчинены нормальному закону с математическим ожиданием равным нулю и среднеквадратическим отклонением равным 5/3\%=1.6667\% от плановых значений. На Рис. 1 приведены значения показателей, полученные в результате моделирования для числа модельных расчетов $N_{\text {_ model }}=155$.

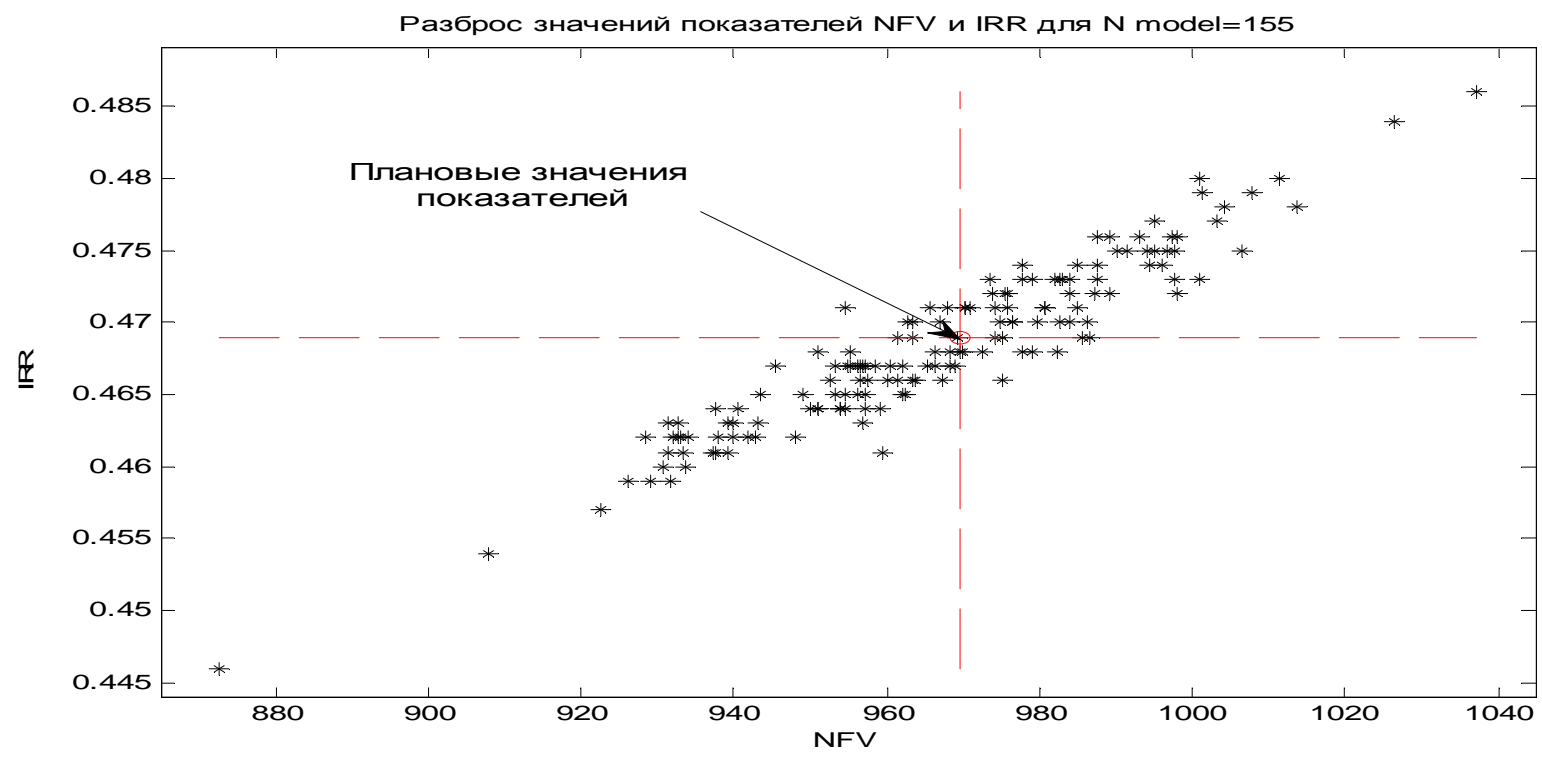

Рисунок 1 - Разброс значений показателей $N F V_{(l)}$ и $I R R_{N F V_{(l)}}$.

Найдем оценку риска в виде наибольших абсолютных потерь [10]: $R_{4, N F V_{(l)}}=N F V_{(l)}-\widehat{N F V}_{(l) \text {,min }}=98,7 ; \quad$ индексы $\quad$ у показателя риска означают принадлежность его к определенному виду $\left(j_{0}=4\right)$, и то, относительно какого показателя риск оценивается - $\left(N F V_{(l)}\right)$, где $N F V_{(l)}$ - плановое значение показателя; $\widehat{N F V}_{(l) \text {,min }}$ - наименьшее значение показателя. Аналогично можно оценить риск для второго показателя $R_{4, I R R_{N E V_{(l)}}}=0,023$ и для размытой функции принадлежности. Относительные риски показателей можно оценить так: $R_{5, N F V_{(l)}}=\left(N F V_{(l)}-\widetilde{N F V}_{(l) \text {,min }}\right) / N F V_{(l)}$ (см. подробнее в [10]).

\section{References:}

1. Zadeh L.A. Fuzzy sets. - Information and Control, 1965, Vol. 8, pp. 338-353. 
2. Bellman R., Zadeh L. Decision-making in a fuzzy environment. - Manag Sci, 1970, Vol. 17, pp. 141-164.

3. Zadeh L.A. From imprecise to granular probabilities. - Fuzzy Set Systems, 2005, Vol. 154, pp. 370-374.

4. Zimmermann H-J. An application-oriented view of modelling uncertainty. - European Journal of Operation Research, 2000, Vol. 122, pp. 190-199.

5. Zimmermann H.-J. Fuzzy Set Theory and Its Applications. - Kluwer Academic Publishers, 2001.

6. Bojadziev G., Bojadziev M. Fuzzy Logic for Business, Finance and Management. Singapore: World scientific, 1997.

7. Turban E., Sharda R., Delen D. Decision Support and Business Intelligence Systems. Prentice Hall, 2010.

8. Hussain O.K., Chang E., Hussain F.K., Dillon T.S. A Fuzzy Aproach to Risk Based Decision Making. - Lecture Notes in Computer Science, Springer Berlin/Heidelberg, Vol. 4278, 2006, pp. 1765-1775.

9. Hillson D. Effective opportunity management for projects - exploiting positive risk. New York: Marcel Dekker, 2004.

10. Наумов А.А. Методы анализа и синтеза инвестиционных проектов. Эффективность, риски, управление. - LAP LAMBERT Academic Publishing, 2013. 356 c. 